\title{
NOTE \\ Internal Medicine \\ A case of canine hypoadrenocorticism needing blood transfusion for severe acute anemia due to gastrointestinal hemorrhage
}

\author{
Yuya KIMURA ${ }^{1) *}$, Sayuri IWAKI ${ }^{2)}$, Satoshi KAMESHIMA ${ }^{1)}$ and Naoyuki ITOH ${ }^{1)}$ \\ 1)Laboratory of Small Animal Internal Medicine, School of Veterinary Medicine, Kitasato University, \\ Higashi 23-35-1, Towada, Aomori 034-8628, Japan \\ ${ }^{2)}$ Misawa Veterinary Treatment Facility, Public Health Activity-Japan, Bldg. 1370 Misawa AB, Misawa, \\ Aomori 033-0022, Japan
}

J. Vet. Med. Sci.

82(1): 31-34, 2020

doi: 10.1292/jvms.19-0075

Received: 4 February 2019

Accepted: 7 November 2019

Advanced Epub:

19 November 2019
ABSTRACT. A 3-year-old male Rottweiler presented with the chief complaint of recurrent vomiting, diarrhea, hypothermia, and lethargy. Hypovolemic shock was noted with abnormal electrolytes ( $\mathrm{Na} / \mathrm{K}$ ratio, 27.9) and anemia (hematocrit, $17.3 \%)$. Since the hematocrit was $49.2 \%$ four days earlier when the primary veterinarian examined the dog, acute anemia was diagnosed. Melena was observed on the next day. The general condition and hydration improved with treatment, and an adrenocorticotropic hormone stimulation test identified hypoadrenocorticism. However, the hematocrit decreased further to $9 \%$, necessitating blood transfusion. The cause of severe acute anemia was thought to be gastrointestinal hemorrhage. It should be noted that hypoadrenocorticism can lead to potentially fatal anemia with gastrointestinal tract bleeding, and blood transfusion may be required.

KEY WORDS: anemia, blood transfusion, gastrointestinal hemorrhage, hypoadrenocorticism, melena

Hypoadrenocorticism (HA), also called Addison's disease, is a relatively rare endocrine disorder, with a reported prevalence of $0.09 \%$ [4]. It is characterized by deficiency of glucocorticoids and mineralocorticoids. As mineralocorticoids regulate serum electrolytes, typical HA is associated with hyponatremia and hyperkalemia. There have been some reports of lymphocytosis and eosinophilia in HA, but other clinicopathological abnormalities are vague and nonspecific. While mild non-regenerative anemia is also a well-known finding (although partly masked by dehydration) [8], concurrent gastrointestinal tract bleeding sometimes aggravates anemia. The mechanism of the bleeding is not fully elucidated. However, glucocorticoid deficiency and hypovolemia can reduce blood flow of gastric mucosal microvessels, resulting in ulceration.

In a retrospective case series reported by Peterson et al. [10], 11 of 225 (4.9\%) dogs with HA and a hematocrit (HCT) lower than $20 \%$ had melena, but the minimum HCT of these dogs was $14.2 \%$. Anemia in HA with or without gastrointestinal tract bleeding seems usually not to be fatal. Although Medinger et al. [9] reported three HA dogs requiring blood transfusion for severe anemia, their gastrointestinal bleeding might have been caused by side effects of previous corticosteroid therapy, and one dog was also treated with a nonsteroidal anti-inflammatory drug. The detailed clinical course of severe (potentially fatal) anemia due to spontaneous gastrointestinal bleeding in HA has not been described. Herein, we report a dog with HA that required blood transfusion due to severe acute anemia, presumably caused by gastrointestinal bleeding. Information about the clinical presentation of this case should be useful for alerting HA with gastrointestinal bleeding.

The patient was a 3-year-old male Rottweiler referred for recurrent vomiting, diarrhea, hypothermia, and lethargy. The symptoms had commenced around 20 days ago and temporarily improved after treatment with antibiotics and fluid therapy by the primary veterinarian, but recurred a few days later. The findings on initial examination were reported to be nonspecific, although electrolyte levels were not assessed. There was no history of excessive exercise or accidental ingestion.

When admitted to Kitasato University Teaching Hospital for detailed examination, the dog weighed $27.8 \mathrm{~kg}$ (body condition score $2 / 5)$. Examination revealed hypothermia $\left(36.6^{\circ} \mathrm{C}\right)$, lethargy, dehydration, pallor of the buccal mucosa, and a delayed capillary refilling time $(5 \mathrm{sec})$. Investigation of the complete blood count and serum chemistry revealed regenerative anemia, leukocytosis, thrombocytopenia, mild hyperglycemia, azotemia, elevated liver enzymes, and electrolyte abnormalities (Table 1). Anemia was thought to be acute, because HCT was $49.2 \%$ when checked four days earlier at the local clinic. The examination of the blood film showed anisocytosis and megathrombocytes. Radiography revealed a small heart (Fig. 1) and fecal impaction from the descending colon to the rectum. The maximum dorsoventral thickness of the left adrenal gland was $4.2 \mathrm{~mm}$ on ultrasound, while the right 
Table 1. Summary of biochemistry and complete blood cell count during hospitalization. The patient received blood transfusion after Day 4 examination, then was discharged on Day 7

\begin{tabular}{|c|c|c|c|c|c|c|}
\hline Parameter & Day 1 & Day 2 & Day 3 & Day 4 & Day 5 & Reference \\
\hline $\operatorname{RBC}\left(10^{6} / \mu l\right)$ & 3.28 & 2.49 & 1.77 & 2.17 & 4.3 & $5.65-8.87$ \\
\hline Hematocrit (\%) & 17.3 & 13.2 & 9.0 & 12.6 & 26.6 & $37.3-61.7$ \\
\hline Hemoglobin $(\mathrm{g} / \mathrm{d} l)$ & 7.0 & 5.2 & 3.6 & 4.5 & 9.4 & $13.1-20.5$ \\
\hline $\operatorname{MCV}(\mathrm{f} l)$ & 52.7 & 53.0 & 54.2 & 58.1 & 61.9 & $61.6-73.5$ \\
\hline $\operatorname{MCHC}(\mathrm{g} / \mathrm{d} l)$ & 40.5 & 39.4 & 37.5 & 35.7 & 35.3 & $32.0-37.9$ \\
\hline Reticulocyte $\left(10^{3} / \mu l\right)$ & 24.3 & 29.1 & 32.7 & 87.5 & 153.5 & $10.0-110.0$ \\
\hline $\mathrm{WBC}(/ \mu l)$ & 32,290 & 21,970 & 16,100 & 24,910 & 30,650 & $5,050-16,760$ \\
\hline Neutrophil $(/ \mu l)$ & 28,400 & 17,050 & 11,600 & 17,220 & 22,780 & $2,950-11,640$ \\
\hline Lymphocyte $(/ \mu l)$ & 2,060 & 3,290 & 3,400 & 6,090 & 6,220 & $1,050-5,100$ \\
\hline Monocyte $(/ \mu l)$ & 1,130 & 590 & 1,020 & 1,500 & 1,550 & $160-1,120$ \\
\hline Eosinophil $(/ \mu l)$ & 610 & 1,020 & 80 & 90 & 70 & $60-1,230$ \\
\hline Basophil $(/ \mu l)$ & 0 & 20 & 0 & 10 & 30 & $0-100$ \\
\hline Platelet $\left(10^{3} / \mu l\right)$ & 67 & 57 & 85 & 109 & 178 & $148-484$ \\
\hline $\mathrm{TP}(\mathrm{g} / \mathrm{d} l)$ & 5.7 & 5.2 & 5.4 & 6.7 & 7.5 & $5.6-7.5$ \\
\hline Albumin $(\mathrm{g} / \mathrm{d} l)$ & 3.1 & 2.8 & 2.9 & 3.6 & 3.9 & $2.9-3.8$ \\
\hline Glucose $(\mathrm{mg} / \mathrm{d} l)$ & 191 & 120 & 126 & - & 108 & $88-128$ \\
\hline $\operatorname{AST}(\mathrm{U} / l)$ & 58 & 76 & 58 & 45 & 42 & $14-42$ \\
\hline $\operatorname{ALT}(\mathrm{U} / l)$ & 240 & 220 & 190 & 200 & 190 & $15-79$ \\
\hline $\operatorname{ALP}(\mathrm{U} / l)$ & 939 & 803 & 655 & 771 & 721 & $50-316$ \\
\hline Direct bilirubin $(\mathrm{mg} / \mathrm{d} l)$ & 0.05 & - & - & - & 0.03 & $0.00-0.06$ \\
\hline Indirect bilirubin $(\mathrm{mg} / \mathrm{d} l)$ & 0.03 & - & - & - & 0.06 & $0.00-0.07$ \\
\hline $\mathrm{BUN}(\mathrm{mg} / \mathrm{d} l)$ & 111.3 & 37.1 & 14.4 & 13.1 & 13.0 & $7.4-27.0$ \\
\hline Creatinine $(\mathrm{mg} / \mathrm{d} l)$ & 1.4 & 0.5 & 0.7 & 0.7 & 0.6 & $0.3-1.2$ \\
\hline $\mathrm{Na}(\mathrm{mmol} / \mathrm{l})$ & 118.1 & 130.7 & 138.7 & 143.8 & 139.6 & $142-152$ \\
\hline $\mathrm{K}(\mathrm{mmol} / l)$ & 4.24 & 5.02 & 4.28 & 5.35 & 5.67 & $3.4-4.8$ \\
\hline $\mathrm{Cl}(\mathrm{mmol} / \mathrm{l})$ & 82.4 & 96.7 & 106.6 & 107.9 & 103.5 & $106-116$ \\
\hline $\mathrm{Na} / \mathrm{K}$ & 27.9 & 26.0 & 32.4 & 26.9 & 24.6 & - \\
\hline
\end{tabular}

RBC, red blood cell; MCV, mean corpuscular volume; MCHC, mean corpuscular hemoglobin concentration; WBC, white blood cell; TP, total protein; AST, aspartate aminotransferase; ALT, Alanine aminotransferase; ALP, alkaline phosphatase; BUN, blood urea nitrogen.

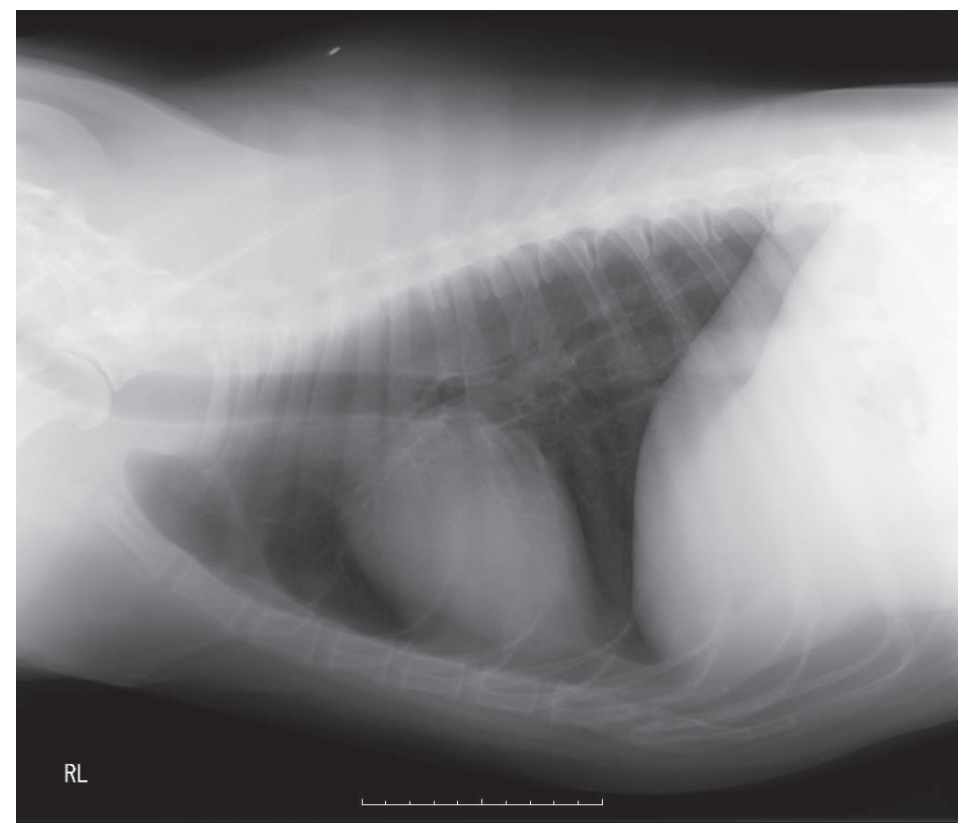

Fig. 1. Thoracic radiograph in the right lateral projection. The small-sized heart suggests a decrease of circulating blood volume. 
adrenal was undetectable. Both imaging methods revealed no pleural or peritoneal effusion or findings suggestive of neoplasia or foreign bodies. Examination of solid yellow feces were retrieved from the anus using a sampling rod showed no intestinal parasites. Urinalysis demonstrated a specific gravity of 1.011 and $\mathrm{pH}$ of 7.1, and was negative for protein, glucose, ketones, and bilirubin. No bacteria grew on culture.

From these findings, hypovolemic shock due to HA and hemorrhage or immune-mediated hemolysis were suspected. HA can present with hypoglycemia [10,13], but it was inferred that hyperglycemia in this case was due to stress causing adrenal crisis [12]. According to Wakayama et al. [13], a normal adrenal gland size does not exclude the diagnosis of HA. Fluid therapy with saline $(8 \mathrm{ml} / \mathrm{kg} / \mathrm{hr})$ was started for hypovolemic shock. The flow rate was reduced to $5 \mathrm{ml} / \mathrm{kg} / \mathrm{hr}$ after 2 hr when the general condition improved slightly, and the dog was able to raise his head after another $2 \mathrm{hr}$. On the next day, the dog showed restoration of vigor and appetite, and administration of single dose of dexamethasone $(0.1 \mathrm{mg} / \mathrm{kg}$, intravenously) led to further improvement, although rehydration highlighted the previously masked hypoproteinemia and severe anemia (Table 1). There was no vomiting or diarrhea during hospitalization. However, melena was observed, and bleeding from the gastrointestinal tract was strongly suspected as the cause of acute anemia. Since blood from the upper gastrointestinal tract takes a certain time to be excreted in the stool, the normal impacted stool collected on Day 1 would have predated the onset of bleeding. In addition to the absence of autoagglutination, spherocytes, and visible mucosal jaundice, a negative Coombs test ruled out immune-mediated hemolytic anemia. Low blood and urine levels of bilirubin also excluded a hemolytic disorder. On Day 4, two days after cessation of dexamethasone for the washout period [7], an adrenocorticotropic hormone (ACTH) stimulation test was performed for diagnosis of HA. The serum cortisol level was measured before and one hour after administration of tetracosactide acetate $(0.25 \mathrm{mg}$, intramuscularly). Both cortisol levels were below the detection limit, confirming the diagnosis of HA. Although melena stopped after the ACTH stimulation test and there was a gradual increase of the mean corpuscular volume and reticulocyte count, blood transfusion (400 $\mathrm{m} l$ ) was performed with dexamethasone premedication $(0.1 \mathrm{mg} / \mathrm{kg}$, intravenously). Following the definitive diagnosis of HA, the dog was discharged on Day 7 with a prescription for fludrocortisone acetate $(0.3 \mathrm{mg} / \mathrm{head}$, twice daily). The $\mathrm{Na} / \mathrm{K}$ ratio was 27 at that time. Treatment was continued by the primary veterinarian, and the $\mathrm{Na} / \mathrm{K}$ ratio on Day 26, 43, 87 and 103 was 26.3, 25.0, 26.3 and 27.6, respectively. The $\mathrm{Na} / \mathrm{K}$ ratio did not respond despite improvement of the general condition, so the regimen was altered. Three months later, when the dog and his family moved to the United States, the dose of fludrocortisone acetate was increased to $0.5 \mathrm{mg} /$ head twice daily, and prednisolone was also administered as needed. Then treatment was changed to almost monthly injections of desoxycorticosterone pivalate $(1.9 \mathrm{mg} / \mathrm{kg}$, subcutaneously). This regimen maintained a stable condition, and the $\mathrm{Na} / \mathrm{K}$ ratio was 36.3 after six months. The HCT was also normal at that time, with a value of $48.7 \%$.

Ulcerations of the digestive tract can occur for various reasons [2]. For the present case, hepatic, renal, pancreatic, or spinal disease and treatment with corticosteroids or nonsteroidal anti-inflammatory drugs could be excluded through interview and the clinical findings. Since endoscopy was not performed, it can be considered a limitation that neoplastic or inflammatory disease of the digestive tract could not be completely denied. However, it was speculated that gastrointestinal bleeding was associated with $\mathrm{HA}$, as the symptoms improved and bleeding did not recur after in initiation of treatment for HA.

Although HA is usually thought to be characterized by mild, non-regenerative anemia, it should be noted that severe acute anemia may occur when concurrent gastrointestinal bleeding exists [10]. In glucocorticoid-suppressed rats, gastric ulceration was associated with decreased gastric blood flow, and was improved by glucocorticoid injection $[3,5]$. These findings suggest that glucocorticoids provide gastric protection by maintaining gastric blood flow. Therefore, it has been hypothesized that reduction of blood flow in the gastric mucosal microvessels by severe hypovolemia and glucocorticoid deficiency leads to gastric ulceration in HA. Also, the present case revealed that such hemorrhagic HA can lead to severe (potentially fatal) anemia in dogs without medications that could precipitate gastrointestinal bleeding, so early detection and treatment are required. The gold standard for exploring the gastrointestinal tract is endoscopy [2]. However, it is impractical owing to the necessity for anesthesia, especially since HA increases vulnerability to stress. Thus, comprehensive clinical assessment is required to confirm gastrointestinal tract bleeding. Melena is generally not a sensitive indicator of gastrointestinal hemorrhage (only observed in 13-50\%), but it is specific [2]. Peterson et al. [10] reported that only $15 \%$ of dogs with HA showed melena, usually on the first or second day of hospitalization, while all dogs with severe anemia (HCT $<20 \%$ ) had concomitant melena. In the present case, the cause of acute anemia was initially unclear, but subsequent detection of melena indicated gastrointestinal tract hemorrhage as the etiology. Therefore, melena seems to be a sensitive and specific finding in hemorrhagic HA, although it might be late. General laboratory findings suggesting hemorrhage include thrombocytopenia and hypoalbuminemia, while azotemia is a characteristic finding in gastrointestinal tract hemorrhage. However, dehydration and circulatory failure can obscure elevation of blood urea nitrogen. The present case showed azotemia, which resolved when hydration was improved. Hence it was uncertain whether the azotemia was caused by circulation failure or the bleeding. Although using peroxidase-based tests to detect fecal occult blood may be useful, results are influenced by the diet $[1,11]$. Elevated liver enzymes were also present, possibly because hepatocytes are vulnerable to anoxia resulting from anemia [6], as is seen in hemolysis.

The present case indicates that it is important to remember that HA may be associated with severe anemia possibly caused by gastrointestinal bleeding. Attention to this risk is also required during treatment, since glucocorticoids may also cause gastrointestinal ulcers and bleeding $[2,5]$. Gastrointestinal hemorrhage should be investigated if HA presents with severe acute anemia and early blood transfusion may need to be considered. 


\section{REFERENCES}

1. Cook, A. K., Gilson, S. D., Fischer, W. D. and Kass, P. H. 1992. Effect of diet on results obtained by use of two commercial test kits for detection of occult blood in feces of dogs. Am. J. Vet. Res. 53: 1749-1751. [Medline]

2. Daure, E., Ross, L. and Webster, C. R. L. 2017. Gastroduodenal ulceration in small animals: part 1. pathophysiology and epidemiology. $J$. Am. Anim. Hosp. Assoc. 53: 1-10. [Medline] [CrossRef]

3. Filaretova, L., Maltcev, N., Bogdanov, A. and Levkovich, Y. 1999. Role of gastric microcirculation in the gastroprotection by glucocorticoids released during water-restraint stress in rats. Chin. J. Physiol. 42: 145-152. [Medline]

4. Hanson, J. M., Tengvall, K., Bonnett, B. N. and Hedhammar, Å. 2016. Naturally occurring adrenocortical insufficiency-an epidemiological study based on a Swedish-insured dog population of 525,028 dogs. J. Vet. Intern. Med. 30: 76-84. [Medline] [CrossRef]

5. Henderson, A. K. and Webster, C. R. L. 2006. Disruption of the gastric mucosal barrier in dogs. Compendium 28: 340-354.

6. Karmaniolou, I. I., Theodoraki, K. A., Orfanos, N. F., Kostopanagiotou, G. G., Smyrniotis, V. E., Mylonas, A. I. and Arkadopoulos, N. F. 2013. Resuscitation after hemorrhagic shock: the effect on the liver-a review of experimental data. J. Anesth. 27: 447-460. [Medline] [CrossRef]

7. Kemppainen, R. J., Sartin, J. L. and Peterson, M. E. 1989. Effects of single intravenously administered doses of dexamethasone on response to the adrenocorticotropic hormone stimulation test in dogs. Am. J. Vet. Res. 50: 1914-1917. [Medline]

8. Klein, S. C. and Peterson, M. E. 2010. Canine hypoadrenocorticism: part I. Can. Vet. J. 51: 63-69. [Medline]

9. Medinger, T. L., Williams, D. A. and Bruyette, D. S. 1993. Severe gastrointestinal tract hemorrhage in three dogs with hypoadrenocorticism. J. Am. Vet. Med. Assoc. 202: 1869-1872. [Medline]

10. Peterson, M. E., Kintzer, P. P. and Kass, P. H. 1996. Pretreatment clinical and laboratory findings in dogs with hypoadrenocorticism: 225 cases (1979-1993). J. Am. Vet. Med. Assoc. 208: 85-91. [Medline]

11. Rice, J. E. and Ihle, S. L. 1994. Effects of diet on fecal occult blood testing in healthy dogs. Can. J. Vet. Res. 58: 134-137. [Medline]

12. Torre, D. M., deLaforcade, A. M. and Chan, D. L. 2007. Incidence and clinical relevance of hyperglycemia in critically ill dogs. J. Vet. Intern. Med. 21: 971-975. [Medline] [CrossRef]

13. Wakayama, J. A., Furrow, E., Merkel, L. K. and Armstrong, P. J. 2017. A retrospective study of dogs with atypical hypoadrenocorticism: a diagnostic cut-off or continuum? J. Small Anim. Pract. 58: 365-371. [Medline] [CrossRef] 